\title{
DISPLACEMENT OF THE CEREBELLUM FROM TUMOUR OF THE POSTERIOR CRANIAL FOSSA. ${ }^{1}$
}

BY WILLIAM G. SPILLER, M.D.

Professor of Neuropathology and Assnciate-Professor of Neurology in the University of Pennsyliania.

I DESIRE to call attention in this paper to two forms of displacement of the cerebellum as a result of tumour of the posterior cranial fossaviz., the lateral displacement, in which the cerebellum is nearly at a right angle with the brain-stem; and the upward displacement, in which the tentorium is much distended and the occipital lobes are widely separated by the dislocated cerebellum.

Displacement of the cerebellum from tumour of the cerebello-pontile angle so that the cerebellum is twisted greatly toward one side is of unusual occurrence. In my collection of nine large tumours external to the cerebellum, in or near the angle mentioned, extreme lateral displacement resulted only twice. I do not include in this number tumours seen at operation, or intracranial tumours that developed in the region of the trigeminal nerve, or tumours in the cerebellum. Usually atrophy of the parts about a tumour is produced when the pressure is intense, and twisting of the cerebellum is therefore less likely to occur.

The twisting of the cerebellum complicates operation for removal of a tumour of the posterior cranial fossa. The medulla oblongata becomes less resistant, and a fatal termination is more likely to follow operation than in cases in which less distortion of the medulla oblongata has occurred. The cerebrospinal fluid also may be increased, as in my Case No. 558, reported in this paper, and the escape of so large an amount of fluid produces very grave symptoms. The twisting of the cerebellum does not necessarily hasten death, although the effect on the vagus nerve from displacement of the medulla oblongata might be regarded as serious.

In Case 1 [No. 421] the symptoms of tumour first appeared about three and a half years before death. They became intense, and

\footnotetext{
1 Read before the Philadelphia Nourological Society, January 27, 1911.
} 
although the displacement of the cerebellum was great, the ateral twisting on the brain-stem was not so extreme as in Case 2 [No. 558], in which the symptoms were much less severe. In Case 1 the tumour was much larger than in Case 2, and it must have stretched the tentorium greatly, as it extended far enough forward to press upon the left oculomotor nerve. It also depressed the left occipital lobe. A deep depression of this part of the brain is a somewhat rare occurrence from

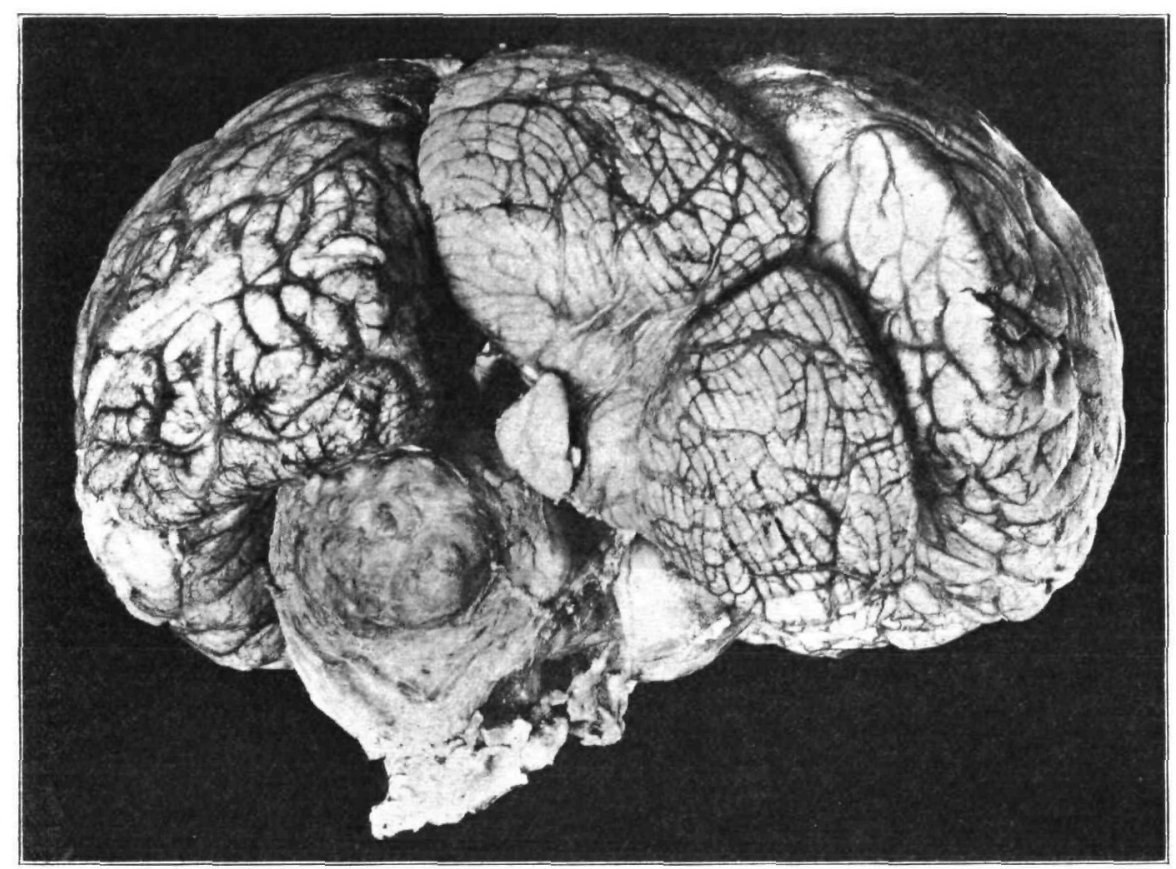

FIG. 1.

Case 1 [No. 421]. - The occipital lobes are widely separated by the displaced cerebellum.

a subtentorial tumour. The displaced cerebellum separated the occipital lobes from one another, and the space provided in this way diminished the degree of lateral twisting of the cerebellum upon its axis. It is possible that during life the separation of the occipital lobes was not so great as is represented in fig. 1, as the tentorium, though greatly distended, must have afforded considerable resistance to pressure. A brief reference to this case with the photograph has been published in a previous paper by $m e$ in the Joumal of the American Medical Association, vol. lii, No. 4, p. 278, 1909.

Two parts of the brain are especially affected by the distortion of 
the cerebellum-viz., the pons at its union with the cerebral peduncles, and the medulla oblongata. If the pressure be from below upward with sufticient force to separate the occipital lobes and stretch the tentorium, as in Case 1, the lateral displacement of the cerebellum is less and the stretching of the medalla oblongata also is less. When the pressure is from one side and displaces the cerebellar lobes almost to a right angle with the brain-stem, as in Case 2, the stretching of the medulla oblongata is pronounced, and this part of the brain may be displaced as

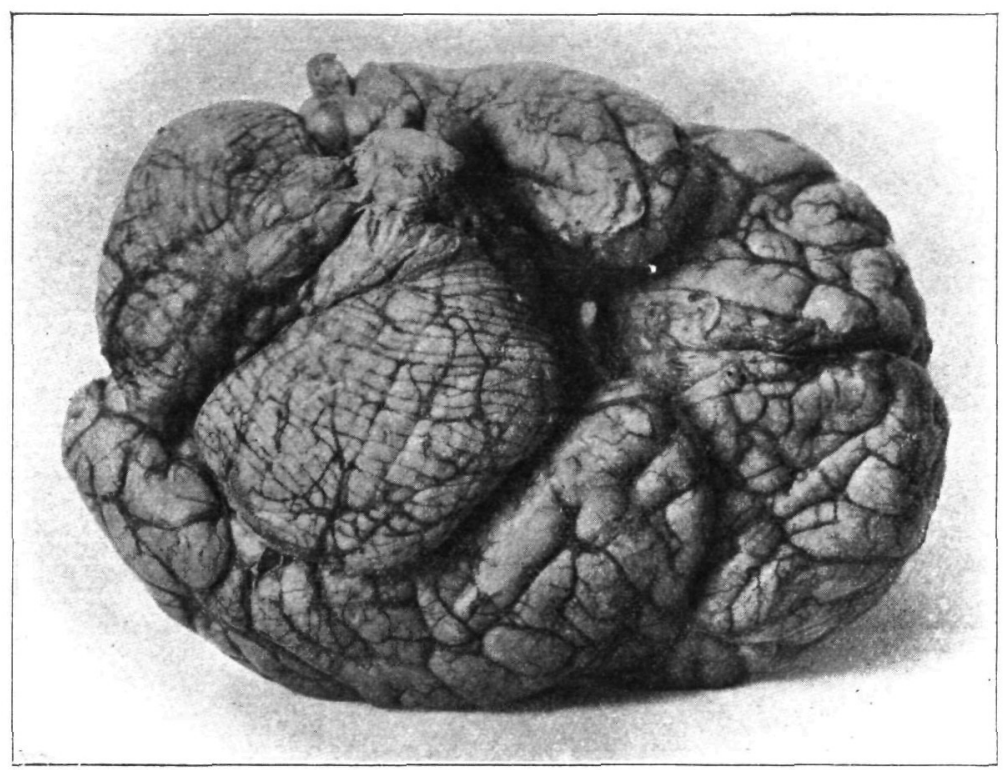

Frg. 2.

Case 2 [No. 558]. - The tumour is on the right side, and has pushed the cerebellum to the left. The pons and medulla oblongata are displaced $3.5 \mathrm{~cm}$. to the right (the side of the tumour) by the left lobe of the cerebellum.

much as $3.5 \mathrm{~cm}$. Inasmuch as the medulla oblongata is continuous with the cervical cord, a dislocation of such a degree would cause stretching of the nerves arising from the former. In Case 2 the cerebellum was pushed to the left by the tumour, but the pons and the medulla oblongata were pushed by the displaced left cerebellar lobe to the right-i.e., to the side of the tumour-and the left side of the pons was much flattened by the pressure from the left cerebellar lobe.

In some instances symptoms have been observed indicative of a 
lesion on the side opposite to that occupied by a basal tumour. An explanation has been sought in pressure upon the medulla oblongata on the side of the tumour, below the motor decussation, or in pressure of the pons and medulla oblongata of the opposite side against the base of the skull, or, more recently by Oppenheim, in the constriction of the medulla oblongata by the tightly stretched vertebral artery. Oppenheim has considered it possible that in lateral displacement of the pons and mednlla oblongata, the pyramidal tract on the convex side of the archi.e., the opposite side to the tumour-may be more damaged. I am not aware that anyone has referred to stretching of cranial nerves on the side opposed to the tumour by the displacement of the pons and medulla oblongata from the median line in the direction of the tumour by a dislocated cerebellar lobe, or to pressure on the contralateral side of the pons by the displaced cerebellar lobe, as seen in Case 2.

The pain in Case 2 was described as unbearable, as of a pressing or burning character, and as though someone were pressing on the side of the head. It was situated in about the left motor region, bat extended well into the left occipital region. Very little pain was felt in the left side of the face. The sensation was more one of burning than of pain. The supra-orbital and infra-orbital foramina were tender to pressure on both sides. In speaking, and voluntary drawing up the corners of the mouth, together or separately, the left side of the face was not moved so much as the right, but the inequality of the movement was slight. Hearing for the voice and watch was considerably impaired in the left ear, though much less than in the right ear.

It is possible that the intense pain in the left side of the head, the slight weakness of the muscles innervated by the left facial nerve, and the moderate degree of deafness in the left ear, were caused by stretching of the left trigeminal, facial, and acoustic nerves from the displacement of the medulla oblongata and pons $3.5 \mathrm{~cm}$. to the right-i.e., toward the side of the tumour. This displacement was produced by pressure from the distorted left lateral lobe of the cerebellum, and is very different to the displacement referred to by Oppenheim, which is to the side opposite to the tumour.

It is uncertain whether headache can be caused by stretching of the trigeminal nerve. It seems at least possible, as the nerves of the cerebral dura, according to "Gray's Anatomy," are filaments from the trochlear, the ophthalmic division of the trigeminal, the semilunar, or Gasserian ganglion, the vagal, the hypoglossal, and the sympathetic. In Case 2, the supra-orbital and infra-orbital formina on each side were 
tender to pressure. The stretching of the left trigeminal nerve may have caused this tenderness on the left side, and the implication of the right trigeminal nerve in the tumour at its surface doubtless caused the tenderness on the right side.

One is impelled to ask why it is that lateral displacement of the cerebellum does not occur more frequently.

In a case of Dr. Mills' [No. 342] the tumour was very much larger than in Case 2, and was in the same situation, but it formed dense adhesions with the left cerebellar lobe and pons, and caused much atrophy of these parts. It did not cause any important displacement of the cerebellum. It was as firm a tumour as that in Case 2, and

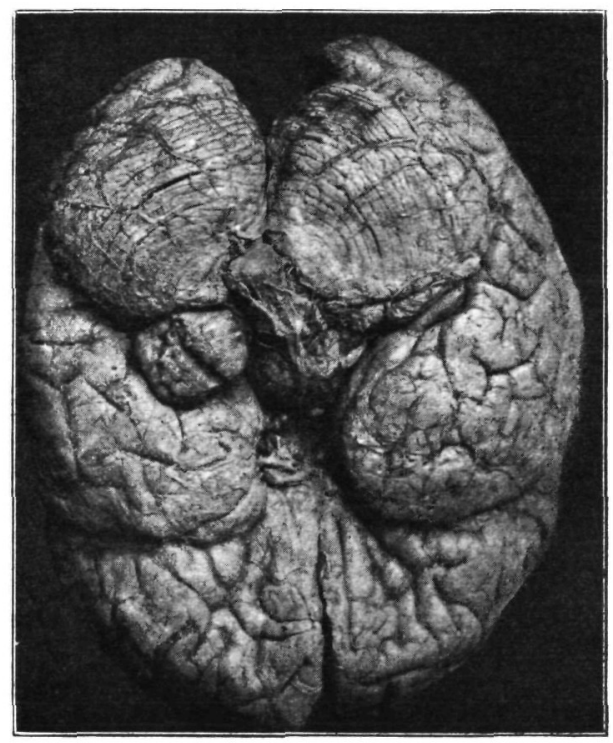

F'so. 3.

Case 3 [No. 205]. -A amall tumour having a similar origin to that in Case 2, but causing no displacement of the cerebellum.

grew in the same direction, along the margin of the cerebellar lobe. It caused moderate atrophy of the overlying occipital lobe. The adhesions of the tumour to the surrounding parts were much stronger than were those of Case 2 , and probably in this fact lies the explanation of the absence of displacement of the cerebellum. The pressure on the cerebellum from so large a tumour (measuring $5.5 \mathrm{~cm}$. antero-posteriorly by $3.5 \mathrm{~cm}$. laterally) must have been greater then from the tamour in Case 2, therefore neither position nor size of the growth is sufficient alone to explain the greater displacement of

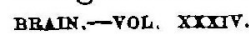


the cerebellum in Case 2, but between these two tumours a difference exists in the density of the adhesions between the tumours and the surrounding parts. If adhesions form early between the tumour and the cerebellum on one side, and the tentorium on the other, the likelihood of much displacement of the cerebellum is lessened, and atrophy of the parts about the tumour will occur. The cerebellum being bound to the tumour by dense adhesions resists displacement, and atrophies proportionately to the pressure from the tumour. Other factors are concerned, however, in the displacement of the cerebellum.

In Case 3 [No. 205], one of 'Dr. Mills', with whom I saw the patient (reference has also been made by Dr. Weisenburg to this case), the tumour was about the size of that in Case 2, was equally firm and equally well defined from the surrounding tissues. It occupied a very similar position, but, unlike the tumour of Case 2, it caused considerable atrophy of the overlying occipito-temporal lobe, this through the tentorium, and only moderate atrophy of the adjoining cerebellar lobe. This tumour measured $2.5 \mathrm{~cm}$. antero-posteriorly by $3 \mathrm{~cm}$. laterally, but where it arose from the cerebello-pontile angle it measured only $1.5 \mathrm{~cm}$. The greater diameter was at its outer portion, where it sent a projection anteriorly which caused pronounced atrophy of the occipito-temporal lobe. The shape of the tumour as well as the atrophy of the occipito-temporal lobe, which could only have been produced by distension of the tentorium, indicates that the pressure from the tumour must have been chiefly forward.

The resistance afforded by the tentorium must be another factor in the displacement of the cerebellum; where the membrane is dense and unyielding, the pressure against the cerebellum is greater.

In Case 4 [No. 338], also one of Dr. Mills', the adhesions between the cerebellum and the tumour were very dense, and great atrophy of the cerebellar lobe on the same side and of this side of the pons occurred. The cerebellom had not been displaced laterally, although this tumour measured $5 \mathrm{~cm}$. antero-posteriorly by $6 \mathrm{~cm}$. laterally by $4 \mathrm{~cm}$. from above downward. The pressure evidently was from below upward, for while only slight atrophy of the overlying occipital lobe had been caused, this occipital lobe had made a deep indentation in the upper part of the lateral lobe of the cerebellum on the side of the tumour by the pressure of the inferior and median edge of the occipital lobe, and the cerebellum had been pushed upward between the two occipital lobes, separating the occipital points as much as $6 \mathrm{~cm}$. The pressure from the displaced cerebellam on the median 
side of each occipital lobe must have been considerable, not so much, however, as in Case 1, where the occipital points were separated as much as $8 \mathrm{~cm}$.

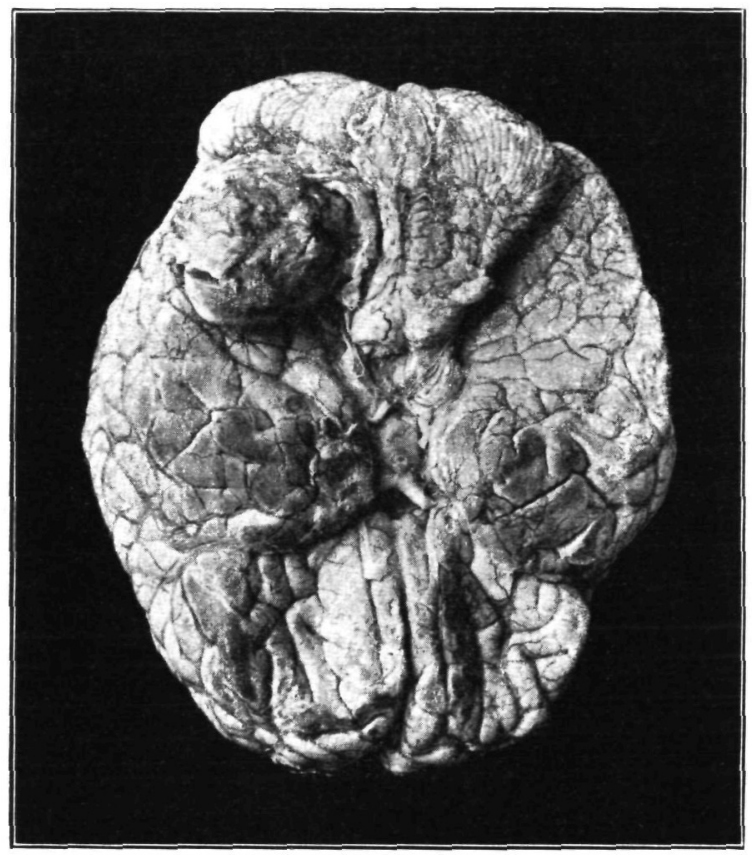

Fig. 4.

Case 4 [No. 398].-A much larger tumour than that in Cage 2, and having a similar origin, but causing little displacement of the cerebellum.

In Case 5 [No. 296] where the patient was under my care the separation was even greater. A large tamour measuring $5 \mathrm{~cm}$. antero-posteriorly by $6 \mathrm{~cm}$. laterally by $5 \mathrm{~cm}$. from above downward, developed in the cerebello-pontile angle, but its origin doubtless was from the dura at the base of the posterior cranial fossa. It was not adherent to the brain. The pressure being from below upward, it caused no lateral displacement of the cerebellum, but it drove the cerebellum far upward between the occipital lobes, separating the occipital points $9 \mathrm{~cm}$. Such displacement as this, notwithstanding the intervening tentorium, gives a fair idea of the great'pressure that must have existed, and suggests the possibility of occipital lobe symptoms from cerebellar tumour. As the displacement, however, cannot occur until the pressure from the tumour becomes great, and not until papillodema has developed, hemianopsia could not be expected, and symptoms of occipital lobe lesion would be masked. 
Condensed notes of the two cases selected as illustrative of the two forms of displacement described in this paper are as follows :-

Case 1 (laboratory number 421).--E. M., female, aged 20, was admitted to the Philadelphia General Hospital, May 1, 1907, and died January 8, 1908. Her sight had been poor since she was 7 years old. Frontal headache had existed about three years, but it had become much worse during the two months before her entrance in the hospital. Two weeks before any notes were taken she began to have difficulty in walking and in speaking. When she came to the hospital she was unable to walk or stand alone, and had a tendency to fall backward, but the voluntary power in the lower limbs was

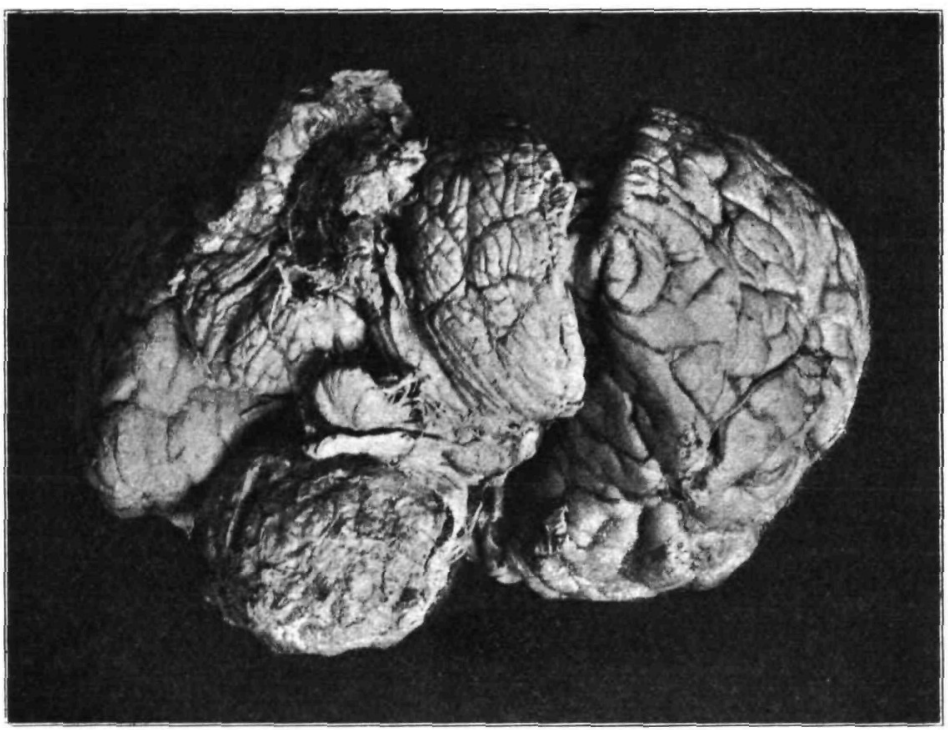

F'J. 5 .

Case 5 [No. 296]. - A tumour of the posterior cranial fossa. It has pushed the cerebellum between the ocoipital lobes as did the tumour in Oase 1.

fair. She was very ataxic even in the heel-to-knee test. The patellar reflexes were exaggerated, and ankle-clonus and the upward movement of the toes in the Babinski test were obtained on each side. The movements of the upper limbs were ataxic, and the grip of each hand was weak. The biceps reflex was exaggerated on each side. The voice was whining. Weakness of the lower part of the right side of the face was present. The tongue deviated to the right when protruded. The right masseter muscle was weak, and the lower jaw deviated slightly to the right when the mouth was opened. These symptoms evidently were caused by pressure upon the left cerebral peduncle and left upper part of the pons. Reaction of the iris to light and in convergence was lost in each eye, and the left eye protruded. Swallowing was difficult. Mentality became much impaired, and stupor pronounced. 
The body was in the cerebellar attitude described by Hughlings Jackson. Dr. Hansell found the late stage of high-grade optic neuritis passing into atrophy in both eyes. The left eyeball could be moved only slightly, and the movement preserved was downward, and slight ptosis was observed on the left side. Late in the disease the right eyeball deviated outward. The right upper limb was a little weeker than the left. Hunger was pronounced, and a second dinner was demanded nearly every day. The upper limbs became very rigid, the fingers were flexed into the palms and the forearms flexed on the arms across the chest. The lower limbs also became very rigid in extension and the feet orer-extended.

A tumour measuring $9 \mathrm{~cm}$. antero-posteriorly by $6 \mathrm{~cm}$. laterally was found in the left cerebello-pontile angle. It was very nodular and well defined. It evidently had arisen from the dura at the base of the brain, and had affected the nerves coming from the left side of the medulla oblongata and pons by pressure only. The cerebellum had been greatly displaced laterally, but not so much as in Case 2, the edge of the right lateral lobe of the cerebellum not so nearly forming a right angle with the brain-stem. The tumour had forced apart the occipital lobes, and bad depressed the basal part of the occipito-temporal lobe. It must have stretched the tentorium greatly. The greater part of the periphery of the tumour, especially the upper part, was covered by a dense fibrous membrane, and a piece of tentorium extended medianly from this membrane. The tumour pressed severely upon the left third nerve, but the right third nerve was not implicated. The posterior and anterior horns of the left lateral ventricle were much compressed. The right lateral ventricle was dilated at all parts. The tumour was a fibro-sarcoma.

Case 2 [laboratory number 558].-S. W., female, aged 33, was seen by me in consultation with Dr. H. D. Beyea, December 22, 1910. She had had serere headache for thirteen months. She had nausea and vomiting occasionally, and her condition had become much worse following an operation for retroflexion of the uterus three weeks previously. The pain in the head was almost unbearable and was of a burning character, and the head felt as though there were internal pressure. This pain was distinctly more intense on the left side, although it extended at times to the right side. Tinnitus like escaping air was heard in the right ear, and hearing was much impaired in the right ear; it appeared to be impaired also in the left ear. Pain had been felt frequently on the right side of the face. She had vertigo at times.

The patellar reflexes were exaggerated. She was ataxic on standing and walking, and the ataxia was increased by closing the eyes. Slight nystagmus was present in looking to the right.

Dr. B. A. Randall reported, December 23: "There is defect of hearing on the right side of moderate degree only for all tones, more marked for the weaker high or low tones. This corresponds with a lesion of the acoustic nerve, but in contradiction she claims transmission through the bone to the affected right ear."

Dr. Langdon, in Dr. de Schweinitz's clinic, on December 23, 1910, found 
3 dioptres of swelling of the optic disc in each eye, and numerous hæemorrhages on and near the discs. On December 27 he found that the papillœdema had increased until the apex of each disc was $5 \frac{1}{2}$ dioptres. In addition, several fresh hæmorrhages had formed.

The reflex of the right scleral conjunctiva and, to a less extent, of the right cornea was diminished. Diadokokinesis was good in each hand. Fingerto-nose test showed slight ataxia only on the right side. Asynergia was moderate; when the patient was told to bend the bead and body backward she at first was not inclined to flex the knees, and nearly fell backward. When told to lift the upper part of the body, with the upper limbs folded across the chest, she was unable to do so at first, as she raised the lower limbs from the bed instead of pressing them against the mattress.

The most serious symptoms, therefore, were nausea, vertico. headache (more on the left side), pain at times in the right side of the face, which was with difficulty to be distinguished from the headache, as it was not present at the time of examination; diminution of the right conjunctival and corneal reflexes; tinnitus and deafness, probably of nerve origin, in the right ear; rapidly developing papillœdema with numerous hæmorrhages; ataxia on standing and walking; exaggeration of the tendon reflexes of the lower limbs, that varied in intensity from one examination to another; ataxia in the finger-tonose test and a moderate amount of asynergia.

My diagnosis was of tumour in the right cerebello-pontile angle, but as the deafness of the right ear was not positively of nerve origin, a tumour of the cerebellum could not be excluded. The right facial nerve showed no defect.

An opening of large size was made over the occipital region by Dr. C. H. Frazier, and the dura was slit. Much cerebrospinal fluid escaped. It was thought advisable to perform the operation in two stages, but the patient did not rally sufficiently to justify any further surgical procedure, and died within a few days.

A tumour, measuring $4 \mathrm{~cm}$. antero-posteriorly by $2.5 \mathrm{~cm}$. laterally, was found in the right cerebello-pontile angle. It was well defined, nodular, and at no part infiltrated the brain. It had displaced the cerebellum almost to a right angle with the brain-stem. The left lateral lobe of the cerebellum indented the pons. The right trigeminal nerve was attached to the tumour at its periphery, but was not involved within the tumour. A bundle of nerve-fibres, having the origin of the acoustic nerve, entered the tumour and was lost within it. It was impossible to determine without dissection whether this was the acoustic or facial nerve, or both, but no trace of any other nerve that could be either of these was found. The tumour pressed upon the right side of the medulla oblongata and flattened this side, and may have stretched the glosso-pharyngeal and vagal nerves. The pressure of the tumour upon the pons and medulla oblongata had not caused any distension of the lateral or of the third ventricle. The tumour was of the same character as that of Case 1, viz., a fibro-sarcoma. Mallory, however, regards these tumours as endotheliomata. 